\title{
Dislocation description of twins in high-temperature superconductor $\mathrm{YBa}_{2} \mathrm{Cu}_{3} \mathrm{O}_{7-\delta}$
}

\author{
V.S. Boyko \\ Physics Department, New York City College of Technology of the City University of New York, Brooklyn, NY, 11201, USA \\ E-mail: vboyko@CityTech.Cuny.Edu \\ Received January 11, 2008
}

\begin{abstract}
The thin twins theory consistently applied to the dislocation description of twins in the high-temperature superconductor $\mathrm{YBa}_{2} \mathrm{Cu}_{3} \mathrm{O}_{7-\delta}(\mathrm{YBCO})$. Quantitative analysis of the twin shape shows an agreement with theoretical consideration and gives new method to evaluate the twin boundary energy $\gamma_{\mathrm{tw}}$. Friction stress $S_{\mathrm{fr}}$ acting on a twinning dislocation is also determined. The formation of a twin and twin microstructures are analyzed. The principles of microstructure design by twinning in YBCO crystals for enhanced $J_{c}$ at high magnetic fields are discussed.
\end{abstract}

PACS: 74.72.Bk Y-based cuprates;

74.25.Qt Vortex lattices, flux pinning, flux creep;

61.72.Lk Linear defects: dislocations, disclinations;

61.72.Mm Grain and twin boundaries.

Keywords: twin boundaries, dislocations, flux pinning.

\section{Introduction}

Mechanical twinning is one of the main forms of plastic deformation of crystals and, because of this, mechanical properties related to twinning have been important materials issues [1,2]. Dislocation description of twining turned out to be very productive. It could be attributed, in particular, to dislocation thin twins theory developed by A.M. Kosevich and his disciples (for details see review [3] and books [4,5]). The development of this theory was initiated by an article of A.M. Kosevich and L.A. Pastur [6] published in 1961. It was a long-term interest in Kharkov scientific community to the investigation of twinning. Exactly 70 years ago, in 1938, R.I. Garber discovered elastic twinning in calcite. Twins in calcite attracted attention of prominent physicists including Huygens, Brewster, Kelvin, and others. In 1890, Voigt found that the calculated strength of calcite with respect to twinning 1000 times greater than real strength. There was no any explanation to this dramatic discrepancy between theory and experiment for abut 40 years. This problem attracted an attention of I.V. Obreimov and he suggested R.I. Garber to work in direction of Voigt problem solution. R.I. Garber developed new technique of twinning and in fine experiments discovered a new phenomenon - elastic twinning. This discovery explained
Voigt paradox and stimulated formation of a twinning dislocation concept. I.M. Lifshitz and I.V. Obreimov as well as T.A. Kontorova and Ya.M. Frenkel analyzed the twinning process at the atomic level. K.V. Vladimirskii used a macroscopic approach. He considered elastic twin as a surface at which there is rapture of stresses and estimated the ratio of twin thickness to twin length. I.M. Lifshitz developed a general macroscopic theory of twins using a nonlinear theory of elasticity [7]. The most important result of K.V. Vladimirskii work was the introduction of the concept of twinning dislocation in 1947 [8]. This concept was independently introduced by Frank and van der Merwe in 1949 [9]. Small value of the ratio of the twin thickness $h$ to its length $L$ allowed to consider all dislocations of an elastic twin as distributed in a single plane (the twinning plane) [10]. In an article of A.M. Kosevich and L.A. Pastur [6], that initiates the thin twins theory, the distribution of twinning dislocations was described by density of dislocations $\rho(x)$ that is the continuous function of coordinate $x$. The condition of equilibrium of a single twinning dislocation in this plane pile-up of twinning dislocations under the action of elastic and inelastic forces used as integral equation for determination of $\rho(x)$ and, as a result, to the description of a twin shape in a medium. In the frame of this approach, A.M. Kosevich and L.A. Pastur considered twins in an unbounded isotropic and 
anisotropic solids, twins perpendicular to the plane surface of isotropic and anisotropic solids and so on [6,11-13]. The development of a twin in the isotropic plane-parallel plate and near the boundary between two anisotropic media was considered by L.A. Pastur, V.S. Boyko, and E.P. Feldman in [14,15]. A.M. Kosevich and L.A. Pastur analyzed in [11] relationship between the thin twins theory and I.M. Lifshitz general macroscopic theory of twins. A.M. Kosevich made two important steps that made possible the direct comparison of the thin twin theory with experimental observations. He considered hysteresis under elastic twinning conditions [16] and introduced phenomenological parameters of the theory and developed approach of determination of the twin length in terms of these parameters [17]. Dynamics of a twin in the frame of the thin twins theory was considered by A.M. Kosevich, V.S. Boyko, and A.A. Slutskin [18-20]. The description of the dynamic motion of the boundary of a residual twin was considered by V.S. Boyko [21]. Thus, all basic stages of the twins development in crystals found adequate theoretical description in the frames of the thin twins theory. Theoretical results of the thin twins theory were in qualitative agreement with experimental observations in calcite made by R.I. Garber, I.V. Obreimov, V.I. Startsev, V.Z. Bengus, E.I. Stepina, and some observations of twinning in metals and alloys made by V.I. Startsev, V.M. Kosevich, V.P. Soldatov, S.V. Lubenets, L.S. Fomenko, I.A. Gindin, V.M. Finkel, V.I. Bashmakov, and others.

V.S. Boyko, R.I. Garber, and L.F. Krivenko $[22,23]$ developed the new experimental methods of formation of thin twins as macroscopic pile-ups of rectilinear twinning dislocations under the influence of distributed load applied accordingly to theoretically calculated external elastic field. In the cycle of quantitative investigations performed by V.S. Boyko, R.I. Garber, L.F. Krivenko, and V.F. Kivshik (for details see [4,5]), the basic results of thin twins theory related to the statics and dynamics of elastic twinning of calcite were experimentally confirmed. As a result, the complete quantitative description of elastic twinning in calcite on the dislocation level was achieved. Generalization of the approach of thin twins theory to the case of pile-ups of transformation dislocations in the external elastic, thermal, electric, and magnetic fields allowed A.M. Kosevich and V.S. Boyko to develop a new unified approach for description of the basic manifestations of reversible plasticity of crystals: elastic twinning, thermoelastic martensitic transformation, superelasticity, shape memory effect, reversible behavior of ferroelastic domains (for details see [4,5,24-26]).

In the twentieth century, most of twin description investigations was dedicated to the plasticity of structural materials by twinning. The thin twins theory turned out to be very productive in this activity. Now, due to microdesign and nanotechnology, the new materials are exploited more and more not as a construction materials but as an essential part of devices. The new materials satisfying the requirements of new technology very often have mechanical properties different from those of traditional materials. As a rule, they have low-symmetry crystal lattices. These materials frequently do not exhibit slip, in contrast to the traditional materials, but do exhibit twinning [27]. Good examples are high- $T_{c}$ superconductors. The detailed analysis of mechanical properties of high- $T_{c}$ superconductors were done in the review [28]. The major problem for industrial applications of high temperature superconductors is their low critical current density $J_{c}$ caused by poor current transmission across high angle grain boundaries (see for details [29]). Contrary to these boundaries, twin boundaries in YBCO improve superconductive properties (see, for example, [30]). Therefore, the description of twin microstructure formation in YBCO has risen as an important problem. Some aspects of applicability of the thin twins theory to the description of twins in YBCO were successfully demonstrated in our joint investigations with group of Professor Chan from Columbia University [31-37]. In this article, we describe the application of the thin twins theory developed mainly for description of mechanical twinning, to the description of the twin structures in the $\mathrm{YBa}_{2} \mathrm{Cu}_{3} \mathrm{O}_{7-\delta}$. The influence of these structures on physical properties of YBCO will be discussed as well.

\section{Shape of a twin}

There is no appreciable mechanical twinning in the $\mathrm{YBa}_{2} \mathrm{Cu}_{3} \mathrm{O}_{7-\delta}$ at conditions of loading material by external elastic field. Therefore the verification of thin twins theory could be done by analysis of the shape of the twins. Consider a lenticular twin column located along the $Y$ axes with the two wedges pointing along $\pm X$ directions for each twin. The $d$ is the distance between the twins along the $Y$ axes. In each individual twin, the negative edge twinning dislocations are located in the region $-L \leq x<0$, while positive edge twinning dislocations are located in the region $0<x \leq L$. The twin is infinitely long along the $Z$ axis, while the thickness of the twin lies along the $Y$ axis. The thickness of the twin $h(x)$ is supposed to be small relatively to the length i.e. $h(0) / L<<1$. In such a case, the twin can be modeled as a pile-up of dislocations in a plane. It is assumed that the distribution of dislocations can be described by a continuous dislocation density function $\rho(x)$ which defines the profile of the twin. In the case of large frictional force and a comparatively long twin, the dislocation density function $\rho(x)$ does not change after twin formation. If the density of dislocations is known, we can determine the shape of the twin by the thickness function: 


$$
h(x)=a \int_{x}^{L} \rho\left(x^{\prime}\right) d x^{\prime},
$$

where $a$ is the interplanar distance of glide planes of twinning dislocations.

There are three regions of a twin along $X$-axis for which meaningful expressions for comparison can be derived [34]. They are the twin tip, the root of twin and the intermediate region. We will discuss each region as follows.

At the twin tip*

$$
h(x)=\frac{4 a(1-v) M}{\mu b \sqrt{L}} \sqrt{L^{2}-x^{2}},
$$

where $b$ is the Burgers vector, $\mu$ is the shear modulus, $v$ is the Poisson ratio, and $S_{\mathrm{st}}(x)$ is the surface tension stress. This stress always tends to shrink the twin and determines $M$

$$
M=\frac{\sqrt{2}}{\pi} \int_{0}^{L} \frac{S_{\mathrm{st}}(x) d x}{\sqrt{L-x}} .
$$

$M$ is a phenomenological parameter of the thin twin theory.

Twin shape in the intermediate region away from the tip is determined by the external stress distribution of $\sigma_{\text {ext }}(x)$ just before unloading. Since this distribution is unknown, we can only qualitatively describe the shape of the twin at the region adjacent to the tip as

$$
h(x) \propto\left[\frac{\pi}{2}-\arcsin \left(\frac{x}{L}\right)-\frac{x}{L} \ln \frac{L+\sqrt{L^{2}-x^{2}}}{x}\right] .
$$

Considering the shape of the twin at the root $(h(x) \sim h(0))$ we should take into account that twins are in lamellae colonies. Central region of the twin could be closer to the neighboring twins than the twin tips. We use a semi-quantitative result [24] to give an approximation and write for the function $h(x)$ at the root of the twin

$$
h(x)=\frac{\pi^{4}(1-v)}{3} \frac{\sigma_{\text {ext }}(0)}{\mu} \frac{a\left(L^{2}-x^{2}\right)}{b d} .
$$

In [34], V.S. Boyko, S.W. Chan, and M. Chopra investigated with electron microscopy the twin shape in $\mathrm{YBa}_{2} \mathrm{Cu}_{3} \mathrm{O}_{7-\delta}$ matrix of melt-textured pellets which contain dispersed particles of $\mathrm{Y}_{2} \mathrm{BaCuO}_{5}$. We compared the recorded images with the shape predicted from the thin twins theory for three regions (twin tip, root and interme- diate region) and found good agreement between the theory and experiment. Thus, the verification of this theory for twin description in $\mathrm{YBa}_{2} \mathrm{Cu}_{3} \mathrm{O}_{7-\delta}$ was done.

\section{Determination of the phenomenological parame- ters of the thin twins theory}

The thin twins theory considers both elastic and inelastic forces acting on twinning dislocations. These forces are incorporated in the thin twin theory as phenomenological parameters $M$ and $S_{\mathrm{fr}}$ - forces of surface tension (i.e. twin boundary tension) and lattice friction (i.e. Peierls force) correspondingly experienced by a single twinning dislocation. If these phenomenological parameters are known, this theory allows to give complete description of mechanical twin behavior including the equilibrium length of a twin and its static shape. Methods for determining these forces were developed in $[22,23]$. They used the data of experimental measurements of the dependences of the elastic twin length on the external applied stress, of a hysteresis of length, of the critical length in bounded crystals. In YBCO crystals, however, all these methods could not be used because the twin length does not change in these crystals after the elastic stresses has been relaxed and as samples were slowly cooled to room temperature. Therefore, we need to develop other approach for determination of phenomenological parameters of the thin twins theory for the new materials in which mechanical twinning does not occur and twin structure is formed during phase transformation. In this case, we can consider and estimate tension force acting on a single twinning dislocation (phenomenological parameter $M$ of the theory) from the shape of a twin. Comparison of the precise twin tip measurement data obtained by electron microscopy measurement and the dislocation thin twins theory predictions (see Sec. 1) opens possibility of the phenomenological parameter $M$ and twin boundary energy $\gamma_{\text {tw }}$ estimate [34]. These twin shape investigations yielded twin boundary energy $\gamma_{\mathrm{tw}}=(60.0 \pm 21.0) \mathrm{mJ} / \mathrm{m}^{2}$. Addition of Pt proves to decrease twin boundary energy to $(26.8 \pm 9.5) \mathrm{mJ} / \mathrm{m}^{2}$ as evaluated by the twin tip shape method. Thus, in [34], V.S. Boyko, S.W. Chan, and M. Chopra suggested a new method (twin tip shape method) of determination of twin boundary energy using electron microscopy measurements of twin tip shape and relating them to the prediction from the dislocation theory of thin twins. This method can be applied to the general case of twin structure when twin tips are observable in addition to the traditional twin

* The thin twins theory predicts a sharp «beak» at the very end of the twin. The size of this «beak» is determined by the action of molecular forces. Therefore this «beak» is very small, and will not be discussed here (possibly, this region could be traced in the observations made by V.S. Boyko, R.I. Garber, A.I. Spol'nik, and L.I. Fedorova in calcite (for details see [5])). 
spacing method (see, for example, [37]). It was also used for investigation of the temperature dependence of $M$ and twin boundary energy in YBCO [39].

In [34], we proposed also method of determination of the second phenomenological parameter of the thin twins theory - frictional force $S_{\text {fr }}$, acting on a single twinning dislocation in YBCO. This quantity can be determined by experimental measurement of a critical plane-parallel twin thickness $h_{c}$ - the smallest thickness of plane parallel twin lamella that can exist in the crystal. The $h_{c}$ is defined [5] as the plane-parallel twin lamella thickness at which the work done against the lattice frictional forces on the twin dislocations during detwinning equals the surface energy of the two twin boundaries. Hence with $h<h_{c}$ twin lamella would disappear while with $h>h_{c}$ twins would be stable. The magnitude of $S_{\text {fr }}$ can be estimated from the relationship:

$$
S_{\mathrm{fr}} \approx \frac{2 a \gamma_{\mathrm{tw}}}{b h_{c}} .
$$

In experiments [34], we did not observe plane-parallel twins thinner than $80 \mathrm{~nm}$ in the crystals without $\mathrm{Pt}$ and plane-parallel twins thinner than $30 \mathrm{~nm}$ in ones with Pt. Substitution value of $\gamma_{t w}$ in equation (5) yielded $S_{\mathrm{fr}} \approx 40 \mathrm{MPa}$ in crystals without $\mathrm{Pt}$ and $S_{\mathrm{fr}} \approx 42 \mathrm{MPa}$ in ones with Pt. $S_{\text {fr }}$ determines the stability of twin microstructure and gives possibility to estimate natural limit of its reduction (see for details [34]). The theoretical consideration and experimental investigations show validity of proposed methods of determination of the twin boundary energy and $S_{\mathrm{fr}}[34,35,39,40]$. After determination of numerical values of phenomenological parameters in $\mathrm{YBCO}$, one can use the dislocation thin twin theory to the full extent. By means of this theory, we will analyze the formation of an individual twin and twin microstructures. The influence of these microstructutes on the superconductive properties of YBCO, will be analyzed as well.

\section{Formation of a twin in the presence of an embedded particle}

Hoping to improve superconductive properties of polycrystal YBCO we can make twin microstructure finer by decrease grain size, but this increases total area of grain boundaries and drops $J_{c}$. The possibility was found to govern twin microstructure large grain YBCO without changing the grain size by incorporating in YBCO matrix the large volume fractions of dispersed addition of (211) in the form of small particles [30,31], dopants [34], and additives such as yttrium nanoparticles [40]. In those cases, the samples were heavily twinned and demonstrate better superconductive properties.
Presence of all these particles embedded in the matrix gives highly nonuniform stress distribution creating twins. The stresses $\sigma_{0}$ corresponding to the appearance of a wedged twin can be estimated (see, for example, [5]) as follows: $\sigma_{0} \approx \gamma_{\mathrm{tw}} / b$. The wedged twin appears in the point of a crystal where this condition is fulfilled. The length of a twin is determined according to [5] by equation

$$
F(L)=\frac{M}{\sqrt{L}}+S_{\mathrm{fr}},
$$

where

$$
F(L)=\frac{2}{\pi} \int_{0}^{L} \frac{\sigma_{e}(x) d x}{\sqrt{L^{2}-x^{2}}},
$$

$\sigma_{e}(x)$ is the stress in the matrix near the immediate vicinity of the embedded particle; $L$ is the length of the twin. Integration is hold along the twin. Twin is originated at the origin of coordinates and is situated along $X$-axis. The real situation is very complicated but qualitative description can be obtained by considering the stress distribution around stiff inclusion. We will use the consideration of elastic equilibrium of a plate with a circular hole in which a circular disk is inserted [41]. When the materials of disk and plate have essentially different elastic constants (it is actually so in our case), we can treat disk to be absolutely rigid. Then, the spatial distribution of the shear stress causing twin appearance can be estimated as

$$
\sigma_{e} \approx \sigma_{0} \frac{a^{2}}{r^{2}},
$$

where $a$ is the radius of the inclusion, $r$ is the distance from the center of inclusion. In the condition of twin formation (comparatively high temperatures and short twins) we can neglect $S_{\mathrm{fr}}$ in (6) that yields $F(L) \approx M / \sqrt{L}$. Using (6), (7), and (8), one can get expression for length of the twin appeared in the vicinity of the inclusion

$$
L \approx\left(\frac{2 a \gamma_{\mathrm{tw}}}{\pi M b}\right)^{2} .
$$

If we take values of data from [34], then for the particle with radius $a \sim 10^{-6} \mathrm{~m}$ we will get $L \sim 10^{-4} \mathrm{~m}$, that correlates by the order of magnitude with the experimental observation made in [32].

\section{Principles of microstructure design by twinning for enhanced critical currents at high magnetic fields}

The influence of twin structures of traditional and high- $T_{c}$ superconductors on their superconductive parameters were discussed in the frames of thin twins theory $[4,5,42,43]$ but with respect to reversible plasticity of su- 
perconductors that may change the ratio of superconductive and nonsuperconductive phases. There is additional possibility of twin microstructure influence on the superconductive properties - the pinning vortices by elements of the twin microstructure. The resources of twin microstructure design in YBCO by twining were analyzed by V.S. Boyko and S.W. Chan in [37] by comparing pinning capability of different microstructures: system of plane-parallel twin lamellas; system of wedgeshaped twins; microstructure composed of twin intersections. The twin intersections are very strong pinning centers $[36,47]$. They tend to pin a fluxoid simultaneously in both directions with the twin boundaries behaving as high-energy barriers, which prevent vortex motion. Such defects possess a very intense elastic field and would tend to suppress the order parameter locally hence improving flux pinning.

Twins in YBCO stem from phase transformation from non-superconducting tetragonal phase to the superconducting orthorhombic phase. In this case, a crystal is penetrated by parallel twin lamellas. Situation was considered using energy approach (see, for example, $[38,44-46])$.The distance between lamellas can be estimated from this approach from a relationship:

$$
T_{w}=\sqrt{\frac{N \gamma_{\mathrm{tw}} R_{\mathrm{col}}}{\mu \varepsilon_{t}^{2}}},
$$

where $T_{w}$ is the distance between centers of lamellas, $R_{\text {col }}$ is the size of the twin colony, $\varepsilon_{t}$ is the twin strain, $\mu$ is the elastic modulus, $N$ is a numerical factor.

The scenario of formation of this type of microstructure could be as follows. The appearance of first twins initiates the appearance of twin colonies around it. The lengths of twins in each colony will increase until they come into a collision with twins of the neighbor colony. Inside each of colony the intertwin distance can be estimated from relationship (10). If each twin intersection can be considered as pinning one vortex, we can estimate trapped magnetic field $H_{\text {tr }}$ by analogy with [48] as follows:

$$
H_{\text {tr }} \sim \phi_{0} \rho_{\text {int }},
$$

where $\phi_{0}$ is elementary magnetic flux $\left(\phi_{0}=2.07 \cdot 10^{-15} \mathrm{~Wb}\right)$, $\rho_{\text {int }}$ - density of twin intersections per unit area. If we assume that $\rho_{\text {int }} \sim\left(T_{w}^{2}\right)^{-1}$, then

$$
H_{\mathrm{tr}} \sim \phi_{0} / T_{w}^{2} \sim \phi_{0} \frac{\mu \varepsilon_{t}^{2}}{N \gamma_{\mathrm{tw}} R_{\mathrm{col}}} .
$$

One can expect that there are two different regimes of formation of intersected areas. In the first regime, concentration of embedded particles is high, and the distance between particles determines the size of a colony. Second regime will be occurred when the concentration of parti- cles is small and the size of colony is smaller than the distance between particles. As a result, $R_{\mathrm{col}} \sim L$ and we will get

$$
H_{\mathrm{tr}} \sim \phi_{0} \frac{\mu^{2} \varepsilon_{t}^{2} b^{2}}{\gamma_{\mathrm{tw}}^{2} a^{2}}
$$

If we take $\varepsilon_{t}^{2} \sim 10^{-3}, \gamma_{\mathrm{tw}} \sim 10^{-2} \mathrm{~J} / \mathrm{m}^{2}, b \sim 10^{-11} \mathrm{~m}$, $\mu \sim 10^{11} \mathrm{~Pa}$ then for the particle with radius $a \sim 10^{-6} \mathrm{~m}$ we will get $H_{\mathrm{tr}} \sim 10 \mathrm{mT} \sim 100 \mathrm{Gs}$, that correlates by the order of magnitude with the experimental data obtained in [47] for the samples with the small concentration of particles. From (13) one can deduce that $H_{\mathrm{tr}} \propto 1 / \gamma_{\mathrm{tw}}^{2}$. Thus we can substantially increase trapped magnetic field $H_{\text {tr }}$ by decreasing twin boundary energy $\gamma_{\mathrm{tw}}$. It is shown in [34] that addition of $\mathrm{PtO}_{2}$ to $\mathrm{YBCO}$ decreases $\gamma_{\mathrm{tw}}$ by two times. Therefore we should expect that samples with $\mathrm{PtO}_{2}$ addition should increase trapped magnetic field by four times in comparison with samples with $\mathrm{CeO}_{2}$ (the addition of $\mathrm{CeO}_{2}$ does not substantially affect the $\gamma_{\mathrm{tw}}$ ). Exactly this enhancement of $H_{\text {tr }}$ was experimentally observed by [49].

Now we will consider regime for which concentration of embedded particles is high, and the size of colony $R_{\mathrm{col}}$ is determined by the distance between particles $R_{p}$ : $R_{\text {col }} \sim R_{p}$. In this case

$$
H_{\mathrm{tr}} \sim \phi_{0} \frac{\mu \varepsilon_{t}^{2}}{\gamma_{\mathrm{tw}} R_{p}} .
$$

If we take $\mu \sim 10^{11} \mathrm{~Pa}, \varepsilon_{t}^{2} \sim 10^{-3}, \gamma_{\mathrm{tw}} \sim 10^{-2} \mathrm{~J} / \mathrm{m}^{2}, R_{p} \sim$ $\sim 10^{-5} \mathrm{~m}, \phi_{0}=2.07 \cdot 10^{-15} \mathrm{~Wb}$ we will get $H_{\mathrm{tr}} \sim 1 \mathrm{~T}$. It is agreed with the estimate of the maximum trapped magnetic field in YBCO containing a completely intersected microstructure at $77 \mathrm{~K}(4.8 \mathrm{~T})$ [47].

Analyzing relationship (14) we can formulate the key ideas of microstructure design for enhanced $J_{c}$ at high magnetic fields. The twin boundary energy and twin colony size are important factors for engineering twin morphology for the strong flux pinning and high $J_{c}$. To improve magnetic properties of YBCO we should investigate possibilities to decrease $\gamma_{\mathrm{tw}}, R_{\mathrm{col}}$. A pinning capability of corresponding basic units of the twin microstructure also should be taken into account (for details see [37]).

\section{Conclusion}

The thin twins theory consistently applied to the dislocation description of twins in the high-temperature superconductor $\mathrm{YBa}_{2} \mathrm{Cu}_{3} \mathrm{O}_{7-\delta}$. The shape of a twin in unloaded medium is determined by the elastic interaction between twinning dislocations and by the inelastic forces (surface tension and friction forces) acting on them. These forces are evaluated by using experimental data of twin shape and relationships of the theory. Quantitative analysis of the twin shape shows an agreement with theo- 
retical consideration and gives new method to evaluate the twin boundary energy $\gamma_{\mathrm{tw}}$. Friction stress $S_{\mathrm{fr}}$ acting on twinning dislocation is also determined. The formation of a twin and twin microstructures are analyzed. The principles of microstructure design by twinning in YBCO crystals for enhanced $J_{c}$ at high magnetic fields are developed.

The stimulating interest and useful comments of L.A. Pastur, V.D. Natsik, and S.V. Lubenets are greatefully aknowledged.

1. M.V. Klassen-Neklyudova, Mechanical Twinnning of Crystals, Consultants Bureau, New York (1964).

2. J.W. Christian and S. Mahajan, Progr. Mater. Sci. 39, 1 (1995).

3. A.M. Kosevich and V.S. Boiko, Sov. Phys. Uspekhi 14, 286 (1971).

4. V.S. Boiko, R.I. Garber, and A.M. Kosevich, Obratimaja Plastichnost» Kristallov, Nauka, Moscow (1991) (in Russian).

5. V.S. Boyko, R.I. Garber, and A.M. Kossevich, Reversible Crystal Plasticity, American Institute of Physics, New York (1994).

6. A.M. Kosevich and L.A. Pastur, Sov. Phys. Solid State 3, 935 (1961).

7. I.M. Lifshitz, Zh. Eksp. Teor. Fiz. 18, 1134 (1948).

8. K.V. Vladimirskii, Zh. Eksp. Teor. Fiz. 17, 530 (1947).

9. F.C. Frank and J.H. van der Merwe, Proc. R. Soc. A198, 205 (1949).

10. V.L. Indenbom, Theory of Twinning, in: the Russian edition of the book (1960), p. 198

11. A.M. Kosevich and L.A. Pastur, Sov. Phys. Solid State 5, 1439 (1964).

12. A.M. Kosevich and L.A. Pastur, Sov. Phys. Solid State 3, 1363 (1961).

13. A.M. Kosevich and L.A. Pastur, Zh. Prikl. Mekh. Tekh. Fiz. 00, 77 (1961).

14. V.S. Boiko, L.A. Pastur, and E.P. Fel'dman, Sov. Phys.Sol. State 8, 2384 (1967).

15. V.S. Boiko and E.P. Fel'dman, Sov. Phys. Solid State 8, 3671 (1967).

16. A.M. Kosevich, Sov. Phys. Solid State 3, 2370 (1962).

17. A.M. Kosevich, Sov. Phys. Solid State 4, 813 (1962).

18. V.S. Boiko, Phys. Status Solidi B55, 477 (1973).

19. V.S. Boiko and A.A. Slutskin, J. Phys. Chem. Sol. 38, 1221 (1977).

20. A.M. Kosevich and V.S. Boyko, Dislocation Theory of the Twinning of Crystals, in: A.M. Kosevich, Dislocations in the Theory of Elasticity, Naukova Dumka, Kiev (1978), p. 127, (in Russian).

21. V.S. Boiko, Sov. Phys. Solid State 22, 621 (1980).

22. V.S. Boiko and R.I. Garber, Sov. Phys. JETP 23, 602 (1966).

23. V.S. Boiko, R.I. Garber, and L.F. Krivenko, Sov. Phys. Solid State 9, 435 (1967).

24. V.S. Boiko, A.M. Kosevich, and E.P. Fel'dman, Sov. Phys. Solid State 29, 94 (1987).
25. V.S. Boyko, A.M. Kosevich, and V.A. Lobodjuk, Russ. Metall. 1, 83 (1992).

26. A.M. Kosevich and V.S. Boiko, Mater. Sci. Eng. A164, 111 (1993).

27. M.H. Yoo and M. Wuttig, Twinning in Advanced Materials, chp. Preface, p. $v$, TMS, Warrendale, PA (1994).

28. S.V. Lubenets, V.D. Natsik, and L.S. Fomenko, Fiz. Nizk. Temp. 30, 467 (2004) [Low Temp. Phys. 30, 345 (2004)].

29. S.-W. Chan, J. Phys. Chem. Solidi 55, 1415 (1994).

30. M. Chopra, S.-W. Chan, R.L. Meng, and C. Chu, J. Mater. Res. 11, 1616 (1996).

31. M. Chopra, V.S. Boyko, R.L. Meng, C.W. Chu, and S.-W. Chan, in: MRS 1995 Fall Meeting, MRS, Boston, MA (1995), p. 48.

32. M. Chopra, S.W. Chan, V.S. Boyko, R.L. Meng, and C.W. Chu, in: Abstracts, 10th Anniversary HTS Workshop on Physics, Materials and Applications, C.W. Chu and K.A. Müller (eds.), Houston, Texas (1996), p. 49.

33. M. Chopra, S.-W. Chan, V.S. Boyko, R.L. Meng, and C.W. Chu, in: Suitable Materials and Processing for HTS Applications: Towards the Next Decade. 1997 International Workshop on Superconductivity, edited by ISTEC-TMS, pp. 311 312, ISTEC MRS, The Royal Waikoloan Big Island, Hawaii, U.S.A. (1997).

34. V.S. Boyko, S.-W. Chan, and M. Chopra, Phys. Rev. B63, 224521 (2001).

35. L. Mei, V.S. Boyko, and S.-W. Chan, Physica C439, 78 (2006).

36. S.-W. Chan, M. Chopra, L. Mei, and V.S. Boyko, in: Program of MRS 2006 Spring Meeting, MRS, San Francisco, CA (2006), p. 230.

37. V.S. Boyko and S.-W. Chan, Physica C466, 56 (2007).

38. T. Roy and T.E. Mitchell, Philos. Mag. A63, 225 (1991).

39. L. Mei and S.-W. Chan, J. Appl. Phys. 98, 033908 (2005).

40. O. Jonprateep and S.-W. Chan, IEEE Trans. Appl. Supercond. 13, 3502 (2003).

41. N.I. Muskhelishvili, Some Basic Problems of the Mathematical Theory of Elasticity, P. Noordhoff Ltd., Groningen (1963).

42. V.S. Boiko and A.M. Kosevich, Fiz. Nizk. Temp. 15, 514 (1989) [Sov. J. Low Temp. Phys. 15, 290 (1989)].

43. V.S. Boiko, A.M. Kosevich, and Yu.A. Kosevich, Fiz. Nizk. Temp. 17, 3 (1991) [Sov. J. Low Temp. Phys. 17, 1 (1991)].

44. D.S. Lieberman, M.S. Wechsler, and T.A. Read, J. Appl. Phys. 26, 473 (1955).

45. A.L. Roytburd, in: Solid State Physics, H. Ehrenreich, F. Seitz, and D. Turnbull (eds.), vol. 33 of Advances in: Research and Applications of Solid State Physics, Academic Press, New York (1978), p. 317.

46. T.E. Mitchell and J.P. Hirth, Acta Metall. Mater. 39, 1711 (1991).

47. M. Chopra, Doctoral Thesis, Columbia University, New York (1997).

48. M.E. McHenry and R.A. Sutton, in: Progress in Materials Science, J.W. Christian and T.B. Massalski (eds.), vol. 38, Pergamon, Oxford New York (1994), p. 159.

49. M.P. Delamare, M. Hervieu, J. Wang, J. Provost, I. Monot, K. Verbist, and G.V. Tendeloo, Physica C262, 220 (1996). 